\title{
Maximum Entropy Approach for Un-Reliable Server Vacation Queueing Model with Optional Bulk Service
}

\author{
Madhu Jain, *G.C. Sharma and **Richa Sharma \\ Department of Mathematics, IIT, Roorkee- 247667 , \\ *Department of Mathematics, I.B.S., Khandari, Agra-282002, \\ **Department of Mathematics, St. Johns College, Agra-282002, India \\ madhufma@iitr.ernet.in
}

\begin{abstract}
In this study, we consider a single server vacation queueing model with optional bulk service and an un-reliable server. A single server provides first essential service (FES) to all arriving customers one by one; apart from essential service, he can also facilitate the additional phase of optional service (OS) in batches of fixed size $b(\geq$ 1 ), in case when the customers request for it. The server may take a single vacation whenever he finds no customers waiting in the queue to be served. Moreover, the server is subjected to unpredictable breakdown while providing the first essential service. The vacation time, service time and repair time of the server are exponentially distributed. The steady state results are obtained in terms of probability generating function for queue size distributions. By using the maximum entropy analysis (MEA), we derive various system performance measures. A comparative study is performed between the exact and approximate waiting time of the system. By taking the numerical illustrations, the sensitivity analysis is done to explore the effect of different descriptors on various performance measures.
\end{abstract}

Keywords: Single vacation, Un-reliable server, Optional batch service, Generating function, Maximum entropy analysis, Queue size, Waiting time.

\section{Introduction}

Queueing models which are characterized by the fact that the server is unavailable for occasional intervals of times are referred as vacation queueing models. Allowing the server to take vacation makes the 
queueing models more realistic and flexible for studying the more robust queueing systems which can be utilized in some fruitful way while analyzing many congestion situations in different frameworks. Vacation queueing models have been studied extensively due to their wide applicability in many areas such as manufacturing, inventory, telecommunication systems, computer communication networks, etc. For detail account on recent past developments, the readers are referred to the survey paper by Doshi ${ }^{[1]}$. Labzovski et al. ${ }^{[2]}$ examined M/G/1 queueing models with single vacation. In connection with the vacation policy, Zhang $^{[3]}$ considered a single server queueing system with two types of server vacations. Further, the steady state behavior of batch arrival queue with Bernoulli vacation schedule under multiple vacation policy was investigated by Choudhury et al. ${ }^{[4]}$. They have done an extensive analysis of the system including queue size and existence of stationary regime. Choudhury ${ }^{[5]}$ studied a single server queue with vacations and two phases of service. Recently, Chae et al. ${ }^{[6]}$ analyzed the continuous-time GI/M/1 queue with single working vacation (SWV). They derived the steadystate distributions for the number of the customers in the system both at arrival and at arbitrary epochs. Very recently, Goswami and Selvaraju ${ }^{[7]}$ considered a discrete-time single-server queueing model with multiple working vacations. They derived various performance measures such as the stationary queue length distribution, waiting time distribution and the distribution of regular busy period, etc.

In many queueing situations, the server renders the service to the customers in batches. The bulk service makes a queueing model more versatile for analyzing the problems of real-world congestion scenario. A wide variety of applications can be seen in many real life congestion situations encountered in transportation, distribution systems, computer and communication networks, etc. Many researchers have discussed the bulk service queueing models in different frameworks. The state dependent bulk service queueing model with accessible and nonaccessible batches was developed by Jain et al. ${ }^{[8]}$. Gupta and Sikdar ${ }^{[9]}$ studied a single server finite- buffer bulk-service queue in which the service is performed in batches of maximum size ' $b$ ' and minimum size ' $a$ '. An M/G/1 batch service queueing model with single vacation was developed by Sikdar and Gupta ${ }^{[10]}$. Al-khedhairi and Tadj ${ }^{[11]}$ investigated the queueing process of a bulk service queueing system under Bernoulli schedule. Gupta and Sikdar ${ }^{[12]}$ examined a finite-buffer batch arrival and 
batch service queue with single and multiple vacations. They have obtained the steady-state distributions of the number of the customers in the queue at service completion, vacation termination, departure, arbitrary and pre-arrival epochs. Recently, a vacation queue with additional optional service in batches was considered by Murugan and Kalyanaraman $^{[13]}$. Lenin et $a l^{[14]}$ investigated the D-BMAP/PH/1/N queues with discrete phase-type group service.

Over the last few decades, queueing models with un-reliable server reflect practical situations in which the server is subjected to unpredictable breakdowns while rendering service to the customers. The concept of un-reliable server is realistic representation of the system because perfect reliable server is virtually non existent in this real world.. The numerous applications motivated us for studying the queueing situations with unreliable server. The study of unreliable server in queueing systems is worthwhile for modeling of many real time systems. Therefore there has been a growing interest in the analysis of unreliable server queueing models which can be developed in the field of distribution systems, manufacturing and production engineering, computer communication industry, inventory systems, etc. Gray et al. ${ }^{[15]}$ studied unreliable server queueing model with vacations. $\mathrm{Ke}^{[16,17]}$ explained the different vacation policies for an $\mathrm{M} / \mathrm{G} / 1$ queueing system with server breakdown. Further, Fiems et al. ${ }^{[18]}$ discussed a queueing system with disruptive and non-disruptive server interruptions. They obtained various performance measures namely the moments of the queue content and waiting times. $\mathrm{M}^{\mathrm{X}} / \mathrm{G} / 1$ retrial queue with an additional second phase of optional service and service interruption was explored by Choudhury et al. ${ }^{[19]}$. A single server unreliable queue was analyzed by Economou and Kapodistria ${ }^{[20]}$ by using the basic $q$-hypergeometric series. Very recently, unreliable Markovian queueing system has been studied by Efrosinin and Winkler ${ }^{[21]}$ to analyze the threshold policy.

Maximum entropy approach is widely applicable to the study of more complicated queueing models having general inter arrival time, retrial time and service time. Wang et al. ${ }^{[22]}$ used the maximum entropy analysis (MEA) to study the M/G/1 queue with server breakdown under $\mathrm{N}$-policy. Ke and $\operatorname{Lin}^{[23]}$ have established the maximum entropy results for the batch arrival $\mathrm{M} / \mathrm{G} / 1$ queueing system with delaying vacations. They derived the approximate formulae for the steady-state probability distributions of the queue length and performed a comparative analysis 
between the approximate results with established exact results. Further, this approach was employed by $\mathrm{Ke}$ and $\operatorname{Lin}^{[24]}$ for analyzing the $\mathrm{M}^{\mathrm{X}} / \mathrm{G} / 1$ queueing system under N-policy with server breakdown. The maximum entropy analysis of the $\mathrm{M}^{\mathrm{X}} / \mathrm{M} / 1$ queueing system with multiple vacations and server breakdown was carried out by Wang et al. ${ }^{[25]}$. Recently, Wang and Huang ${ }^{[26]}$ have made the comparison between the derived approximate results and exact analytical results for $\mathrm{M} / \mathrm{G} / 1$ queue with unreliable server by using the MEA.

The main objective of present investigation is to explore various aspects of 'bulk service queues' used in wide variety of congestion situations wherein the server serves the optional demands of customers in batches of fixed size. An attempt has been made to incorporate more realistic assumptions of an unreliable server, vacation and additional phase of optional service. A single unreliable server renders the first essential service (FES) singly according to first come first served (FCFS) rule. An additional phase of optional service is also provided by the server in batches of fixed size $(b \geq 1)$ only to those customers who requests for it. The maximum entropy analysis (MEA) is employed to analyze various system characteristics in terms of known mean operational characteristics. The generating function is used to obtain queue size distribution. The organization of the rest of the paper is as follows. In section 2, we describe the model by stating requisite assumptions and notations. The steady state equations of the model are constructed. In next section 3 , the probability generating function method is used to obtain the queue size and closed form expression of the probability of the system being empty. Various performance measures are established in section 4. Some special cases are deduced in section 5 by setting appropriate values of system parameters. The approximate results for the queue size distribution and expected waiting time by using the principle of maximum entropy are obtained in section 6 . Numerical illustrations are facilitated to validate the analytical results in section 7 . The noble features and future scope of the model proposed are outlined at the end of the paper in section 8 .

\section{Model Description}

We consider a single unreliable server queueing model with vacation. The assumptions made to formulate the mathematical model 
are as follows. The customers arrive according to Poisson process with rate $\lambda$. A single server renders first essential service to all arriving customers; it also provides an additional phase of optional service in bulk of fixed size $b(\geq 1)$. It is assumed that the customers are served exponentially with mean $1 / \mu_{1}$ (in first essential service) and $1 / \mu_{2}$ (in optional service). After the completion of first essential service, the customer has an option to leave the system with probability q or may go for optional service with probability $p$. If the batch of fixed size $b(\geq 1)$ is accumulated in the system then the server immediately provides the optional service. After the completion of additional phase of optional service, the server may again provide the first essential service if there are some customers waiting in the queue. Otherwise, the batch having less than $b$ customers can be chosen for optional service if there is no customer waiting in the queue for first essential service.

After the completion of services i.e. first essential service and optional service, the server may take vacation of random length. On returning from vacation if it finds one or more customers waiting in the queue, it serves those customers; otherwise will wait in the system for new arrival. The vacation time of the server follows the exponential distribution with mean $1 / \theta$. There is a random interruption in service due to server failure. The server while rendering first essential service to any customer, may fail in Poisson fashion with rate $\alpha$. Thus the 'active breakdown' of the server during first essential service is considered. The failed server immediately sent for repairing with rate $\beta$ and after the completion of repair, the server works as good as before failure.

The steady state probabilities of the system states are defined as follows:

$\mathrm{P}_{0,0,0}$ : Probability that the server is idle.

$\mathrm{Q}_{\mathrm{m}, 0}$ : Probability that the server is on vacation and $\mathrm{m}(>0)$ customers waiting for the first essential service.

$\mathrm{P}_{\mathrm{m}, \mathrm{n}, 1}$ : Probability that the server is rendering first essential service and $\mathrm{m}(>0)$ and $\mathrm{n}(0<n \leq b-1)$ customers are waiting in the queue for the first essential service and optional service, respectively .

$\mathrm{P}_{\mathrm{m}, 0,2}$ : Probability that the server is rendering optional service and $\mathrm{m}(>0)$ customers are waiting for the first essential service. 
$\mathrm{R}_{\mathrm{m}, \mathrm{n}, 3}$ : Probability that the server is under repair and $\mathrm{m}(>0)$ and $\mathrm{n}($ $0<n \leq b-1)$ customers are waiting in the queue for the first essential service and optional service, respectively .

Using the state transition diagram depicted in Fig. 1, the steady state equations governing the model are given as follows:

$$
\begin{gathered}
\left(\lambda+\mu_{1}+\alpha\right) P_{m, n, 1}=\lambda P_{m-1, n, 1}+\mu_{1} q P_{m+1, n, 1}+\mu_{1} p P_{m+1, n-1,1}+\beta R_{m, n, 3}, m>0,0<n \leq b-1 \\
\left(\lambda+\mu_{1}+\alpha\right) P_{m, 0,1}=\lambda P_{m-1,0,1}+\mu_{1} q P_{m+1,0,1}+\mu_{2} P_{m+1,0,2}+\beta R_{m, 0,3}+\theta Q_{m+1,0}, m>0 \\
\left(\lambda+\mu_{1}+\alpha\right) P_{0, n, 1}=\mu_{1} q P_{1, n, 1}+\mu_{1} p P_{1, n-1,1}+\beta R_{0, n, 3}, \quad 0<n<b \\
\left(\lambda+\mu_{1}+\alpha\right) P_{0,0,1}=\mu_{1} q P_{1,0,1}+\mu_{2} P_{1,0,2}+\beta R_{0,0,3}+\theta Q_{1,0}+\lambda P_{0,0,0} \\
\left(\lambda+\mu_{2}\right) P_{m, 0,2}=\lambda P_{m-1,0,2}+\mu_{1} p P_{m, b-1,1}, m>0 \\
\left(\lambda+\mu_{2}\right) P_{0,0,2}=\mu_{1} p \sum_{n=1}^{b-1} P_{0, n, 1} \\
(\lambda+\theta) Q_{m, 0}=\lambda Q_{m-1,0}, m>0 \\
(\lambda+\theta) Q_{0,0}=\mu_{1} q P_{0,0,1}+\mu_{2} P_{0,0,2} \\
\lambda P_{0,0,0}=\theta Q_{0,0} \\
(\lambda+\beta) R_{m, n, 3}=\alpha P_{m, n, 1}+\lambda R_{m-1, n, 3}, m>0,0<n \leq b-1 \\
(\lambda+\beta) R_{m, 0,3}=\alpha P_{m, 0,1}+\lambda R_{m-1,0,3}, m>0 \\
(\lambda+\beta) R_{0, n, 3}=\alpha P_{0, n, 1}, 0<n<b \\
(\lambda+\beta) R_{0,0,3}=\alpha P_{0,0,1}
\end{gathered}
$$

The normalizing condition is given by

$$
P_{0,0,0}+\sum_{m=0}^{\infty} Q_{m, 0}+\sum_{m=0}^{\infty} \sum_{n=0}^{b-1} P_{m, n, 1}+\sum_{m=0}^{\infty} P_{m, 0,2}+\sum_{m=0}^{\infty} \sum_{n=0}^{b-1} R_{m, n, 3}=1
$$

\section{Probability Generating Function}

In order to obtain the queue size distribution, we define the probability generating functions for the different states of the server as follows: 


$$
\begin{array}{ll}
P_{n, 1}(z)=\sum_{m=0}^{\infty} P_{m, n, 1} z^{m}, P_{m, 1}(y)=\sum_{n=0}^{b-1} P_{m, n, 1} y^{n}, & |z| \leq 1,|y| \leq 1 \\
P_{1}(z, y)=\sum_{m=0}^{\infty} P_{m, 1}(y) z^{m}=\sum_{n=0}^{b-1} P_{n, 1}(z) y^{n}=\sum_{m=0}^{\infty} \sum_{n=0}^{b-1} P_{m, n, 1} z^{m} y^{n}, & |z| \leq 1,|y| \leq 1 \\
P_{0,2}(z)=\sum_{m=0}^{\infty} P_{m, 0,2} z^{m}, Q_{0}(z)=\sum_{m=0}^{\infty} Q_{m, 0} z^{m}, & |z| \leq 1,|y| \leq 1 \\
R_{n}(z)=\sum_{m=0}^{\infty} R_{m, n, 3} z^{m}, R_{m}(y)=\sum_{n=0}^{b-1} R_{m, n, 1} y^{n}, & |z| \leq 1,|y| \leq 1 \\
R(z, y)=\sum_{m=0}^{\infty} R_{m}(y) z^{m}=\sum_{n=0}^{b-1} R_{n}(z) y^{n}=\sum_{m=0}^{\infty} \sum_{n=0}^{b-1} R_{m, n, 3} z^{m} y^{n}, & |z| \leq 1,|y| \leq 1
\end{array}
$$

Multiplying equations (1) and (3) by appropriate powers of $\mathrm{z}$ and summing over $\mathrm{m}$, we obtain

$$
\begin{array}{r}
{\left[\left(\lambda(1-z)+\mu_{1}+\alpha\right) z-\mu_{1} q\right] P_{n, 1}(z)=\mu_{1} p P_{n-1,1}(z)-\mu_{1} q P_{0, n, 1}-\mu_{1} p P_{0, n-1,1}+\beta z R_{n}(z),} \\
0<n \leq b-1
\end{array}
$$

Similarly, multiplying equations (2) and (4) by appropriate powers of $\mathrm{z}$ and summing over $\mathrm{m}$, we get

$$
\begin{aligned}
{\left[\left(\lambda(1-z)+\mu_{1}+\alpha\right) z-\mu_{1} q\right] P_{0,1}(z)=} & \mu_{2} P_{0,2}(x)+\theta Q_{0}(z)-\mu_{1} q P_{0,0,1}-\mu_{2} P_{0,0,2}-\theta Q_{0,0}+\lambda z P_{0,0,1}(16) \\
& +\beta x R_{0}(z),
\end{aligned}
$$

Again, after multiplying equations (5) and (6) by appropriate powers of $\mathrm{z}$ and then summing over $0<n \leq b-1$, we have

$$
\left[\lambda(1-z)+\mu_{2}\right] P_{0,2}(z)=\mu_{1} p P_{b-1,1}(z)+\mu_{1} p \sum_{n=1}^{b-2} P_{0, n, 1}
$$

Similarly, multiplying equations (7) and (8), (10) and (12), (11) and (13) respectively, by appropriate powers of $\mathrm{z}$ and summing over $\mathrm{m}$ and $\mathrm{n}$, we find

$$
\begin{gathered}
{[\lambda(1-z)+\theta] Q_{0}(z)=(\lambda+\theta) Q_{0,0}} \\
{[(\lambda(1-z)+\beta) z] R_{n}(z)=\alpha z P_{n, 1}(z), \quad 0<n \leq b-1} \\
{[(\lambda(1-z)+\beta) z] R_{0}(z)=\alpha z P_{0,1}(z)}
\end{gathered}
$$


Now, multiplying equation (15) by appropriate powers of $\mathrm{y}$ and summing over $0<n \leq b-1$, then adding into equation (16) and doing some manipulation, we obtain

$$
\begin{aligned}
& {\left[\mu_{2} P_{0,2}(z)+\theta Q_{0}(z)-(\lambda+\theta) Q_{0,0}-\lambda(1-z) P_{0,0,0}-\mu_{1}(q+p y) P_{0,1}(y)\right.} \\
P_{1}(z, y)= & \left.+\mu_{1} q P_{0,0,1}+\beta z R(z, y)\right]
\end{aligned}
$$

We notice that $\mathrm{P}_{1}(\mathrm{z}, \mathrm{y})=\mathrm{P}_{0,1}(\mathrm{z}), \mathrm{R}(\mathrm{z}, \mathrm{y})=\mathrm{R}_{0}(\mathrm{z})$ and $\mathrm{P}_{0,1}(0)=\mathrm{P}_{0,0,1}$. Thus for $\mathrm{y}=0$, equation (21) becomes as

$$
P_{0,1}(z)=\frac{\left[\mu_{2} P_{0,2}(z)+\theta Q_{0}(z)-(\lambda+\theta) Q_{0,0}-\lambda(1-z) P_{0,0,0}+\beta z R_{0}(z)\right]}{\left[\left(\lambda(1-z)+\mu_{1}+\alpha\right) z-\mu_{1} q\right]}
$$

For $b=1$, equation (17) gives

$$
P_{0,2}(z)=\frac{\mu_{1} p P_{0,1}(z)}{\left[\lambda(1-z)+\mu_{2}\right]}
$$

From equation (20), we have

$$
P_{0,3}(z)=\frac{\alpha z P_{0,1}(z)}{[(\lambda(1-z)+\beta) z]}
$$

Substituting results given by equations (18) and (23) in equation (22) and simplifying, we have

$$
P_{0,1}(z)=\frac{\lambda\left[\lambda(z-1)-\mu_{2}\right][\lambda(z-1)-\beta]\left[(\lambda+\theta) Q_{0,0}+(\lambda(z-1)+\theta) P_{0,0,0}\right]}{[\lambda(z-1)-\theta] \Omega}
$$

From equations (23), (24) and (25), we get

$$
\begin{array}{r}
P_{0,2}(z)=\frac{\lambda \mu_{1} p[\lambda(z-1)-\beta]\left[(\lambda+\theta) Q_{0,0}+(\lambda(z-1)+\theta) P_{0,0,0}\right]}{[\lambda(1-z)+\theta] \Omega} \\
R_{0}(z)=\frac{\lambda \alpha z\left[\lambda(z-1)-\mu_{2}\right]\left[(\lambda+\theta) Q_{0,0}+(\lambda(z-1)+\theta) P_{0,0,0}\right]}{[\lambda(1-z)+\theta] \Omega}
\end{array}
$$

where

$$
\begin{aligned}
\Omega= & {\left[\lambda^{3} z^{3}-\lambda z^{2}\left\{2 \lambda^{2}+\lambda\left(\alpha+\beta+\mu_{1}+\mu_{2}\right)+\alpha \beta\right\}+z\left[\lambda^{3}+\lambda^{2}\left(\alpha+\beta+\mu_{1}+\mu_{2}+\mu_{1} q\right) .\right.\right.} \\
& \left.\left.+\lambda\left(\beta+\mu_{2}\right)\left(\mu_{1}+\alpha\right)+\beta \mu_{2}(\lambda+\alpha)\right]-\mu_{1}\left(\lambda q+\mu_{2}\right)(\lambda+\beta)\right]
\end{aligned}
$$

Theorem 1: The probability $\mathrm{P}_{0,0,0}$ of the system being empty is 


$$
P_{0,0,0}=\frac{\theta^{2}\left[1-\frac{\lambda}{\mu_{1}}\left(\frac{p \mu_{1}}{\mu_{2}}+1\right)-\frac{\alpha}{\mu_{1}}\left(\frac{\lambda}{\beta}+1\right)\right]}{\left[\theta^{2}+\lambda(\lambda+\theta)-\frac{\alpha}{\mu_{1}}\left(\theta^{2}+\lambda(\lambda+\theta \beta)\right)\right]}
$$

Proof: The probability $\mathrm{P}_{0,0,0}$ can be determined by using normalizing condition. For this purpose, we evaluate $\mathrm{Q}_{0}(1), \mathrm{P}_{0,1}(1), \mathrm{P}_{0,2}$ (1) and $\mathrm{R}_{0}$ (1) from equations (18), (25), (26) and (27), respectively. Thus

$$
\begin{gathered}
Q_{0}(1)=\frac{\lambda(\lambda+\theta)}{\theta^{2}} P_{0,0,0} \\
P_{0,1}(1)=\frac{\lambda \mu_{2}\left[\lambda(\lambda+\theta)+\theta^{2}\right] P_{0,0,0}}{\theta^{2}\left[\mu_{1}\left(\mu_{2}+\lambda q\right)-\lambda\left(\mu_{1}+\mu_{2}\right)-\frac{\alpha}{\beta} \mu_{2}(\lambda+\beta)\right]} \\
P_{0,2}(1)=\frac{\mu_{1} p}{\mu_{2}} P_{0,1}(1) \\
R_{0}(1)=\frac{\alpha}{\beta} P_{0,1}(1)
\end{gathered}
$$

Using the normalizing condition given in equation (14) and substituting the values of $\mathrm{Q}_{0}(1), \mathrm{P}_{0,1}(1), \mathrm{P}_{0,2}(1)$ and $\mathrm{R}_{0}(1)$ from equations (29)-(32), respectively, we obtain the value of initial state probability $\mathrm{P}_{0,0,0}$ as given in (28).

Theorem 2: The probability generating function of the number of the customers in the queue is

$$
G_{q}(z)=\frac{\Omega_{1}\left[\frac{\lambda}{\theta}(\lambda+\theta)+(\lambda(z-1)+\theta)\right] P_{0,0,0}}{\Omega(\lambda(z-1)+\theta)}
$$

where

$$
\begin{aligned}
& \Omega_{1}=\left[\lambda^{3}\left(z^{3}-3 z^{2}+3 z-1\right)-\lambda^{2}\left[z^{2}\left(\beta+\mu_{1}+\mu_{2}\right)-z\left(\beta+\alpha\left(\mu_{1}+\mu_{2}\right)\right)+\left(\mu_{1}+\mu_{2}\right)-\beta(z-1)\right]\right. \\
&\left.-\lambda\left[z^{2} \alpha \beta-z\left(\alpha \beta+2 \beta \mu_{2}+\mu_{1} \mu_{2}\right)+\mu_{1} \mu_{2}+\beta\left(\mu_{1}+\mu_{2}\right)\right]+z \alpha \beta \mu_{2}-\mu_{1} \mu_{2} \beta\right]
\end{aligned}
$$


Proof: The probability generating function of the number of the customers in the queue is obtained as

$$
G_{q}(z)=P_{0,0,0}+Q_{0}(z)+P_{0,1}(z)+P_{0,2}(z)+R_{0}(z)
$$

Substituting the values of $\mathrm{Q}_{0}(\mathrm{z}), \mathrm{P}_{0,1}(\mathrm{z}), \mathrm{P}_{0,2}(\mathrm{z})$ and $\mathrm{R}_{0}(\mathrm{z})$ from equations (18), (25), (26) and (27), respectively in equation (34) and after doing some algebraic manipulations, we obtain the value of $G_{q}(z)$.

\section{Performance Characteristics}

In this section, we establish some useful performance indices by using the long run probabilities obtained in the previous section as given below:

$$
\begin{gathered}
P_{V} \equiv Q_{0}(1)=\frac{\lambda(\lambda+\theta) P_{0,0,0}}{\theta^{2}} \\
P_{E} \equiv P_{0,1}(1)=\frac{\lambda \mu_{2}\left[\lambda(\lambda+\theta)+\theta^{2}\right] P_{0,0,0}}{\theta^{2}\left[\mu_{1}\left(\mu_{2}+\lambda q\right)-\lambda\left(\mu_{1}+\mu_{2}\right)-\frac{\alpha}{\beta} \mu_{2}(\lambda+\beta)\right]} \\
P_{O} \equiv P_{0,2}(1)=\frac{\lambda \mu_{1} \mu_{2} p\left[\lambda(\lambda+\theta)+\theta^{2}\right] P_{0,0,0}}{\theta^{2} \mu_{2}\left[\mu_{1}\left(\mu_{2}+\lambda q\right)-\lambda\left(\mu_{1}+\mu_{2}\right)-\frac{\alpha}{\beta} \mu_{2}(\lambda+\beta)\right]} \\
P_{D} \equiv R_{0}(1)=\frac{\alpha \lambda \mu_{2}\left[\lambda(\lambda+\theta)+\theta^{2}\right] P_{0,0,0}}{\beta \theta^{2}\left[\mu_{1}\left(\mu_{2}+\lambda q\right)-\lambda\left(\mu_{1}+\mu_{2}\right)-\frac{\alpha}{\beta} \mu_{2}(\lambda+\beta)\right]}
\end{gathered}
$$

Theorem 3: The average number of the customers in the queue is given by

$$
E\left(N_{q}\right)=\frac{\left[N^{\prime}(1) D(1)-N(1) D^{\prime}(1)\right]}{[D(1)]^{2}} P_{0,0,0}
$$

where

$$
N(1)=\left(\frac{\lambda}{\theta}(\lambda+\theta)+\theta\right) \Omega_{1}(1) ; D(1)=\theta \Omega(1)
$$




$$
\begin{aligned}
N^{\prime}(1)= & \left(\frac{\lambda}{\theta}(\lambda+\theta)+\theta\right) \Omega_{1}^{\prime}(1)-\lambda \Omega_{1}(1) ; D^{\prime}(1)=\theta \Omega^{\prime}(1)-\lambda \Omega(1) \\
\Omega_{1}(1)= & {\left[\lambda^{2}\left(\mu_{1}+\mu_{2}\right)(\alpha-2)+\lambda \beta\left(\mu_{2}-\mu_{1}\right)+\beta \mu_{2}\left(\alpha-\mu_{1}\right)\right] } \\
\Omega_{1}^{\prime}(1)= & {\left[\lambda^{2}\left(\mu_{1}+\mu_{2}\right)(\alpha-2)+\lambda\left(\mu_{1} \mu_{2}+2 \beta \mu_{2}-\alpha \beta\right)+\alpha \beta \mu_{2}\right] } \\
\Omega(1)= & {\left[\lambda \alpha \mu_{2}+\lambda \beta \mu_{2}+\beta \alpha \mu_{2}+\lambda \beta \mu_{1} p-\beta \mu_{1} \mu_{2}\right] } \\
\Omega^{\prime}(1)= & {\left[\lambda\left(\alpha \mu_{2}+\beta \mu_{1}+\mu_{1} \mu_{2}\right)+\beta \mu_{2}(\lambda+\alpha)-2 \lambda \alpha \beta-\lambda^{2} \mu_{1} p-\lambda^{2}\left(\alpha+\beta+\mu_{2}\right)\right] } \\
& -\lambda\left[\lambda^{2}\left(\mu_{1}+\mu_{2}\right)(\alpha-2)+\lambda \beta\left(\mu_{2}-\mu_{1}\right)+\beta \mu_{2}\left(\alpha-\mu_{1}\right)\right]
\end{aligned}
$$

Proof: Using $E\left(N_{q}\right)=\left.\frac{d G_{q}(z)}{d z}\right|_{z=1}$, we can easily compute the average number of the customers in the queue which is given by equation (39).

Theorem 3: The average waiting time of the customers in the queue is

$$
W_{q}=\frac{\left[N^{\prime}(1) D(1)-N(1) D^{\prime}(1)\right]}{\lambda[D(1)]^{2}} P_{0,0,0}
$$

Proof: To obtain the average waiting time of a customer in the queue, we use the Little's formula given as

$$
W_{q}=\frac{E\left(N_{q}\right)}{\lambda}
$$

Putting the value of $\mathrm{E}\left(\mathrm{N}_{\mathrm{q}}\right)$ from equation (39) in equation (40), we get the value of $\mathrm{W}_{\mathrm{q}}$ as given in equation (40).

\section{Special Cases}

In this section, we deduce some special cases by setting appropriate parameters as follows:

Case 1: A reliable queue with vacation and additional optional service in batches.

On setting $\alpha=0$ and $\beta=0$, our model reduces to the model considered by Murugan and Kalyanaraman ${ }^{[13]}$. 
Case 2: Reliable M/M/1 queue with additional optional service and without single vacation.

By setting $\theta=0, \alpha=0$ and $\beta=0$, we obtain the same results as obtained by Madan ${ }^{[27]}$.

Case 3: A reliable queue without vacation and without additional optional service.

In this case, substituting $\theta=0 \mathrm{p}=0, \alpha=0$ and $\beta=0$, our model reduces to classical M/M/1 model (cf. Gross and Harris ${ }^{[28]}$ ).

\section{Maximum Entropy Analysis}

In order to estimate the probability distribution of an unreliable server $\mathrm{M} / \mathrm{M} / 1$ queue with vacation and additional phase of optional service in bulk, we formulate maximum entropy results by using several known constraints in terms of performance characteristics derived in previous section 4 .

Following El-Affendi and Kouvatsos ${ }^{[29]}$, the entropy function $\mathrm{H}$ can be mathematically formulated as

$$
H=-\sum_{n=0}^{\infty} Q_{0}(n) \log Q_{0}(n)-\sum_{n=0}^{\infty} P_{0,1}(n) \log P_{0,1}(n)-\sum_{n=0}^{\infty} P_{0,2}(n) \log P_{0,2}(n)-\sum_{n=0}^{\infty} R_{0}(n) \log R_{0}(n)
$$

The maximum entropy results are obtained by maximizing entropy function $\mathrm{H}$ given in equation (42) subject to the following constraints:

$$
\begin{aligned}
& \text { (i) } \sum_{n=0}^{\infty} Q_{0}(n)+\sum_{n=0}^{\infty} P_{0,1}(n)+\sum_{n=0}^{\infty} P_{0,2}(n)+\sum_{n=0}^{\infty} R_{0}(n)=1 \\
& \text { (ii) } \sum_{n=0}^{\infty} P_{0,1}(n)=P_{0,1}(1) \equiv a_{1} \\
& \text { (iii) } \sum_{n=0}^{\infty} P_{0,2}(n)=P_{0,2}(1) \equiv a_{2} \\
& \text { (iv) } \sum_{n=0}^{\infty} R_{0}(n)=R_{0}(1) \equiv a_{3}
\end{aligned}
$$




$$
\text { (v) } \sum_{n=0}^{\infty} n Q_{0}(n)+\sum_{n=0}^{\infty} n P_{0,1}(n)+\sum_{n=0}^{\infty} n P_{0,2}(n)+\sum_{n=0}^{\infty} n R_{0}(n)=E\left(N_{q}\right)
$$

Here $a_{1}, a_{2}, a_{3}$ and $\mathrm{E}\left(\mathrm{N}_{\mathrm{q}}\right)$ are known as these can be determined by using equations (36), (37), (38) and (39), respectively.

Now we construct the Lagrange's function $\mathrm{L}\left\{\mathrm{Q}_{0}(\mathrm{n}), \mathrm{P}_{0,1}(\mathrm{n}), \mathrm{P}_{0,2}(\mathrm{n})\right.$, $\left.\mathrm{R}_{0}(\mathrm{n})\right\}$ in order to determine the maximum value of entropy function as

$$
\begin{aligned}
& \mathrm{L}\left\{\mathrm{Q}_{0}(\mathrm{n}), \mathrm{P}_{0,1}(\mathrm{n}), \mathrm{P}_{0,2}(\mathrm{n}), \mathrm{R}_{0}(\mathrm{n})\right\} \\
= & -\sum_{n=0}^{\infty} Q_{0}(n) \log Q_{0}(n)-\sum_{n=0}^{\infty} P_{0,1}(n) \log P_{0,1}(n)-\sum_{n=0}^{\infty} P_{0,2}(n) \log P_{0,2}(n)-\sum_{n=0}^{\infty} R_{0,3}(n) \log R_{0}(n) \\
- & \theta_{1}\left[\sum_{n=0}^{\infty} Q_{0}(n)+\sum_{n=0}^{\infty} P_{0,1}(n)+\sum_{n=0}^{\infty} P_{0,2}(n)+\sum_{n=0}^{\infty} R_{0}(n)-1\right]-\theta_{2}\left[\sum_{n=0}^{\infty} P_{0,1}(n)-a_{1}\right]-\theta_{3}\left[\sum_{n=0}^{\infty} P_{0,2}(n)-a_{2}\right] \\
- & \theta_{4}\left[\sum_{n=0}^{\infty} R_{0}(n)-a_{3}\right]-\theta_{5}\left[\sum_{n=0}^{\infty} n Q_{0}(n)+\sum_{n=0}^{\infty} n P_{0,1}(n)+\sum_{n=0}^{\infty} n P_{0,2}(n)+\sum_{n=0}^{\infty} n R_{0}(n)-E\left(N_{q}\right)\right]
\end{aligned}
$$

where $\theta_{i}(1 \leq i \leq 5)$ are the Lagrange's multipliers corresponding to the constraints (43)-(47), respectively.

The probability distributions based on maximum entropy principle can be obtained by taking the partial derivatives of L w.r. to $\mathrm{Q}_{0}(\mathrm{n})$, $P_{0,1}(n), P_{0,2}(n), R_{0}(n),(n \geq 0)$ respectively, and then setting these results equal to zero. Thus, we obtain

$$
\begin{array}{r}
\frac{\partial L}{\partial Q_{0}(n)}=-\log Q_{0}(n)-1-\theta_{1}-\theta_{5} n=0 \\
\frac{\partial L}{\partial P_{0,1}(n)}=-\log P_{0,1}(n)-1-\theta_{1}-\theta_{2}-\theta_{5} n=0 \\
\frac{\partial L}{\partial P_{0,2}(n)}=-\log P_{0,2}(n)-1-\theta_{1}-\theta_{3}-\theta_{5} n=0 \\
\frac{\partial L}{\partial R_{0}(n)}=-\log R_{0}(n)-1-\theta_{1}-\theta_{4}-\theta_{5} n=0
\end{array}
$$

From equations (49)-(52), we obtain

$$
\begin{aligned}
& Q_{0}(n)=e^{-\left(1+\theta_{1}\right)} e^{-\theta_{5} n}, \quad n \geq 0 \\
& P_{0,1}(n)=e^{-\left(1+\theta_{1}+\theta_{2}\right)} e^{-\theta_{5} n}, \quad n \geq 0
\end{aligned}
$$




$$
\begin{array}{cc}
P_{0,2}(n)=e^{-\left(1+\theta_{1}+\theta_{3}\right)} e^{-\theta_{5} n}, \quad n \geq 0 \\
R_{0}(n)=e^{-\left(1+\theta_{1}+\theta_{4}\right)} e^{-\theta_{5} n}, \quad n \geq 0
\end{array}
$$

Let $\phi_{1}=e^{-\left(1+\theta_{1}\right)}, \phi_{i}=e^{-\theta_{i}}, 2 \leq i \leq 5$

Equations (53)-(56) can be transformed in terms of $\phi_{i}(1 \leq i \leq 5)$ as

$$
\begin{aligned}
& Q_{0}(n)=\phi_{1} \phi_{5}^{n}, \quad n \geq 0 \\
& P_{0,1}(n)=\phi_{1} \phi_{2} \phi_{5}^{n}, \quad n \geq 0 \\
& P_{0,2}(n)=\phi_{1} \phi_{3} \phi_{5}^{n}, \quad n \geq 0 \\
& R_{0}(n)=\phi_{1} \phi_{4} \phi_{5}^{n}, \quad n \geq 0
\end{aligned}
$$

On substituting the values of $\mathrm{Q}_{0}(\mathrm{n}), \mathrm{P}_{0,1}(\mathrm{n}), \mathrm{P}_{0,2}(\mathrm{n})$ and $\mathrm{R}_{0}(\mathrm{n})$ from equations (57)-(60) into equations (43)-(46), we have

$$
\begin{gathered}
\sum_{n=0}^{\infty} \phi_{1} \phi_{5}^{n}=\frac{\phi_{1}}{1-\phi_{5}}=1-\left(a_{1}+a_{2}+a_{3}\right) \\
\sum_{n=0}^{\infty} \phi_{1} \phi_{2} \phi_{5}^{n}=\frac{\phi_{1} \phi_{2}}{1-\phi_{5}}=a_{1} \\
\sum_{n=0}^{\infty} \phi_{1} \phi_{3} \phi_{5}^{n}=\frac{\phi_{1} \phi_{3}}{1-v_{5}}=a_{2} \\
\sum_{n=0}^{\infty} \phi_{1} \phi_{4} \phi_{5}^{n}=\frac{\phi_{1} \phi_{4}}{1-\phi_{5}}=a_{3}
\end{gathered}
$$

It follows from equations (61)-(64) that

$$
\begin{gathered}
\phi_{1}=(1-\Delta)\left(1-\phi_{5}\right) \\
\phi_{2}=\frac{a_{1}}{(1-\Delta)} \\
\phi_{2}=\frac{a_{2}}{(1-\Delta)} \\
\phi_{4}=\frac{a_{3}}{(1-\Delta)}
\end{gathered}
$$

where $\Delta=a_{1}+a_{2}+a_{3}$. 
On substituting the values of $\phi_{1}, \phi_{2}, \phi_{3}$ and $\phi_{4}$ from equations (65)(68) into equation (47) and after doing some algebraic manipulations, we obtain

$$
\phi_{5}=\frac{E\left(N_{q}\right)}{1+E\left(N_{q}\right)}
$$

On substituting the values of $\phi_{1}, \phi_{2}, \phi_{3}$ and $\phi_{4}$ from equations (65)(68) into equations (57)-(60) and using equation (69), we finally get

$$
\begin{aligned}
Q_{0}(n) & =\left(\frac{1-\Delta}{1+E\left(N_{q}\right)}\right)\left(\frac{E\left(N_{q}\right)}{1+E\left(N_{q}\right)}\right)^{n}, \quad n \geq 0 \\
P_{0,1}(n) & =\left(\frac{a_{1}}{1+E\left(N_{q}\right)}\right)\left(\frac{E\left(N_{q}\right)}{1+E\left(N_{q}\right)}\right)^{n}, \quad n \geq 0 \\
P_{0,2}(n) & =\left(\frac{a_{2}}{1+E\left(N_{q}\right)}\right)\left(\frac{E\left(N_{q}\right)}{1+E\left(N_{q}\right)}\right)^{n}, \quad n \geq 0 \\
R_{0}(n) & =\left(\frac{a_{3}}{1+E\left(N_{q}\right)}\right)\left(\frac{E\left(N_{q}\right)}{1+E\left(N_{q}\right)}\right)^{n}, \quad n \geq 0
\end{aligned}
$$

Theorem 4: The approximate expected waiting time in the queue is given by

$$
\hat{W}_{q}=\left[\frac{\left(\mu_{1}+\mu_{2}\right)-a_{1} \mu_{1}-a_{2} \mu_{2}}{\mu_{1} \mu_{2}}\right] E\left(N_{q}\right)+\frac{1-a_{1}-a_{2}-a_{3}}{\theta}+\frac{a_{3}}{\beta}
$$

Proof: To obtain the approximate expected waiting time of the tagged customer ' $\mathrm{C}$ ', we assume that the tagged customer ' $\mathrm{C}$ ' finds $\mathrm{n}$ customers waiting in the queue in front of him to receive their service from the server. The server may be in any one of the following states:

(i) Vacation State $(\mathbf{V})$ : When the arbitrary customer ' $C$ ' finds that the server is on vacation state then he will be served after the remaining vacation time of the server, plus the service time of those $n$ customers waiting in front of him for their service. So, in this state the mean waiting time of arbitrary customer ' $\mathrm{C}$ ' is 


$$
\hat{W}_{V}=\sum_{n=0}^{\infty}\left[\frac{n}{\mu_{1}}+\frac{n}{\mu_{2}}+\frac{1}{\theta}\right] Q_{0}(n)
$$

(ii) Busy State (B): In this state, the server may be busy in providing FES and OS in batches. So in this case arbitrary customer ' $\mathrm{C}$ ' only waits with $\mathrm{n}$ customers in front of him to be served. Thus the mean waiting time of the customer ' $\mathrm{C}$ ' at state $\mathrm{B}$ is

$$
\hat{W}_{B}=\sum_{i=1}^{2} \sum_{n=0}^{\infty} \frac{n}{\mu_{i}} P_{0, i}(n)
$$

(iii) Repair State (R): When the server is in repair state, the waiting time of ' $\mathrm{C}$ ' includes the remaining repair time of the server, service time of $\mathrm{n}$ customers waiting in the queue. Thus the expected waiting time of customer ' $\mathrm{C}$ ' is

$$
\hat{W}_{R}=\sum_{n=0}^{\infty}\left[\frac{n}{\mu_{1}}+\frac{n}{\mu_{2}}+\frac{1}{\beta}\right] R_{0}(n)
$$

On combining the above three results (75)-(77), we obtain

$$
\hat{W}_{q}=\sum_{n=0}^{\infty}\left[\frac{n}{\mu_{1}}+\frac{n}{\mu_{2}}+\frac{1}{\theta}\right] Q_{0}(n)+\sum_{i=1}^{2} \sum_{n=0}^{\infty} \frac{n}{\mu_{i}} P_{0, i}(n)+\sum_{n=0}^{\infty}\left[\frac{n}{\mu_{1}}+\frac{n}{\mu_{2}}+\frac{1}{\beta}\right] R_{0}(n)
$$

Substituting the values of $\mathrm{Q}_{0}(\mathrm{n}), \mathrm{P}_{0,1}(\mathrm{n}), \mathrm{P}_{0,2}(\mathrm{n})$ and $\mathrm{R}_{0}(\mathrm{n})$ from equations (70)-(73) into equation (78), we obtain the approximate waiting time in the queue as given in equation (74).

\section{Numerical Illustrations}

In this section, we have performed numerical experiment by taking an example of doctor clinic to examine the effect of different parameters on the performance characteristics and the average queue length by using 'MATLAB' software. The comparison between the exact waiting time $\left(\mathrm{W}_{\mathrm{q}}\right)$ and the approximate waiting time $\left(\hat{W}_{q}\right)$ of the customers is facilitated. For this purpose, we obtain the absolute percentage error (APE) between $\mathrm{W}_{\mathrm{q}}$ and $\hat{W}_{q}$. The doctor serves each patient individually according to first come first served (FCFS) discipline. The doctor facilitates the essential service i.e. physiotherapy by massage or by other 
means (e.g. electronic equipment). After getting preliminary treatment, some patient may opt for physical exercise (i.e. Yoga)/ meditation that can be considered as second phase optional service which was done in a batch of fixed size under the supervision of doctor. The doctor may not be available due to some personal reason (i.e. unreliable) and also go for a vacation after providing optional service if there is no patient in the clinic.

For illustration purpose, we assume that patients arrive in Poisson fashion with rate $\lambda=0.8 / \mathrm{hr}$. Then, the doctor provides first essential treatment with rate $\mu_{1}=6 / \mathrm{hr}$. After first primary treatment, the patient has an option to join yoga/meditation class with probability $p=0.2$. The service of yoga/meditation class is provided by the doctor with rate $\mu_{2}=5 / \mathrm{hr}$ in batches of fixed size. The doctor may be unavailable with rate $\square=0.5 / \mathrm{hr}$ and returns after some time with rate $\beta=0.7 / \mathrm{hr}$. After providing both of the services, the doctor may take a single vacation and returns from the vacation with rate $\theta=1 \mathrm{hr} /$ day. The expected number of patients in the queue is found to be $\mathrm{E}\left(\mathrm{N}_{\mathrm{q}}\right)=1.66$ and the average waiting time of the patients in the queue is $\mathrm{W}_{\mathrm{q}}=2.08 \mathrm{hr}$.

To illustrate the effect of different parameters on various performance measures, numerical results have been summarized in Tables 1-5 and also shown graphically in Fig. 1-3. We fix the default parameters as $p=0.2, \lambda=0.8, \mu_{1}=6, \mu_{2}=5, \theta=1.2, q=0.8, \alpha=0.5, \beta=0.7$, for Tables 1-3; $p=0.5, \lambda=1.25, \mu_{1}=2, \mu_{2}=1.9, \theta=0.95, q=0.5, \alpha=0.15, \beta=0.17$ for Tables 4-5; $\mathrm{p}=0.2, \lambda=3, \mu_{1}=4, \mu_{2}=3, \theta=0.8, \mathrm{q}=0.8, \alpha=4, \beta=5$ for Fig. $2-4$.

\section{(a) Effect of Arrival Rate and Vacation Rate on Long Run Probabilities}

Table 1 shows the effect of $\lambda$ and $\theta$ on the performance characteristics of the system for the two different values of $p$. It is clear form the table that $\mathrm{P}_{\mathrm{E}}, \mathrm{P}_{\mathrm{O}}$ and $\mathrm{P}_{\mathrm{D}}$ show the increasing trend while $\mathrm{P}_{\mathrm{V}}$ decreases by increasing the values of $\lambda$ and $p$. As the value of $\theta$ increases, $P_{V}, P_{E}, P_{O}$ and $P_{D}$ decrease. On increasing the values of $p$, $\mathrm{P}_{\mathrm{V}}\left(\mathrm{P}_{\mathrm{O}}\right)$ decreases (increases). Moreover, $\mathrm{P}_{\mathrm{E}}$ and $\mathrm{P}_{\mathrm{D}}$ remain almost constant for increasing values of $p$. 


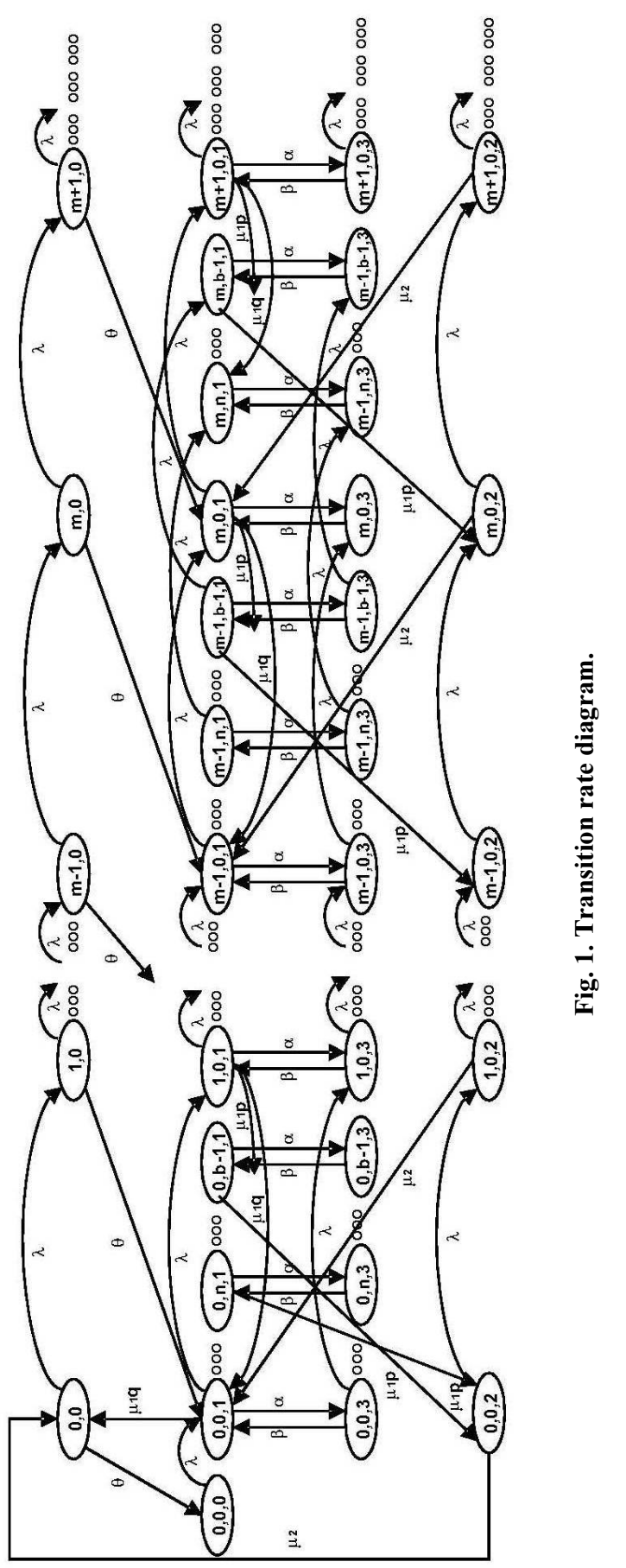


Table 1. Effect of $p, \lambda$ and $\theta$ on various performance characteristics.

\begin{tabular}{||c||c||c|c|c|c||c||c|c|c|c||}
\hline \hline $\mathbf{p}$ & $\lambda$ & $\mathbf{P}_{\mathbf{V}}$ & $\mathbf{P}_{\mathbf{E}}$ & $\mathbf{P}_{\mathbf{O}}$ & $\mathbf{P}_{\mathbf{D}}$ & $\boldsymbol{\theta}$ & $\mathbf{P}_{\mathbf{V}}$ & $\mathbf{P}_{\mathbf{E}}$ & $\mathbf{P}_{\mathbf{O}}$ & $\mathbf{P}_{\mathbf{D}}$ \\
\hline \multirow{4}{*}{.1} & 0.5 & 0.619 & 0.182 & 0.021 & 0.130 & 0.92 & 0.926 & 0.296 & 0.035 & 0.212 \\
\cline { 2 - 11 } & 0.6 & 0.580 & 0.184 & 0.022 & 0.132 & 0.99 & 0.787 & 0.263 & 0.031 & 0.187 \\
\cline { 2 - 10 } & 0.7 & 0.548 & 0.189 & 0.022 & 0.135 & 1.06 & 0.677 & 0.235 & 0.028 & 0.168 \\
\cline { 2 - 10 } & 0.8 & 0.518 & 0.195 & 0.023 & 0.139 & 1.13 & 0.589 & 0.213 & 0.025 & 0.152 \\
\cline { 2 - 10 } & 0.9 & 0.490 & 0.202 & 0.024 & 0.144 & 1.20 & 0.518 & 0.195 & 0.023 & 0.139 \\
\hline \hline \multirow{4}{*}{.3} & 0.5 & 0.603 & 0.182 & 0.065 & 0.130 & 0.92 & 0.882 & 0.296 & 0.106 & 0.212 \\
\cline { 2 - 10 } & 0.6 & 0.561 & 0.184 & 0.066 & 0.132 & 0.99 & 0.749 & 0.263 & 0.094 & 0.187 \\
\cline { 2 - 10 } & 0.7 & 0.526 & 0.189 & 0.068 & 0.135 & 1.06 & 0.645 & 0.235 & 0.084 & 0.168 \\
\cline { 2 - 10 } & 0.8 & 0.493 & 0.195 & 0.070 & 0.139 & 1.13 & 0.561 & 0.213 & 0.076 & 0.152 \\
\cline { 2 - 9 } & 0.9 & 0.463 & 0.202 & 0.072 & 0.144 & 1.20 & 0.493 & 0.195 & 0.070 & 0.139 \\
\hline \hline
\end{tabular}

\section{(b) Effect of Service Rates on Long Run Probabilities}

Table 2 depicts the behavior of $\mu_{1}$ and $\mu_{2}$ on $\mathrm{P}_{\mathrm{V}}, \mathrm{P}_{\mathrm{E}}, \mathrm{P}_{\mathrm{O}}$ and $\mathrm{P}_{\mathrm{D}}$ by varying ' $p$ '. On increasing the values of $\mu_{1}$ and $\mu_{2}, P_{V}$ increases but reverse effect is observed for $\mathrm{P}_{\mathrm{E}}, \mathrm{P}_{\mathrm{O}}$ and $\mathrm{P}_{\mathrm{D}}$.

Table 2. Effect of $p, \mu_{1}$ and $\mu_{2}$ on various performance characteristics.

\begin{tabular}{||c|c||c|c|c|c||c||c|c|c|c||}
\hline \hline $\mathbf{p}$ & $\mu_{1}$ & $\mathbf{P}_{\mathbf{V}}$ & $\mathbf{P}_{\mathbf{E}}$ & $\mathbf{P}_{\mathbf{O}}$ & $\mathbf{P}_{\mathbf{D}}$ & $\boldsymbol{\mu}_{2}$ & $\mathbf{P}_{\mathbf{V}}$ & $\mathbf{P}_{\mathbf{E}}$ & $\mathbf{P}_{\mathbf{O}}$ & $\mathbf{P}_{\mathbf{D}}$ \\
\hline \multirow{4}{*}{.1} & 3.0 & 0.312 & 0.439 & 0.026 & 0.313 & 1.0 & 0.468 & 0.195 & 0.117 & 0.139 \\
\cline { 2 - 11 } & 3.5 & 0.376 & 0.363 & 0.025 & 0.259 & 1.3 & 0.483 & 0.195 & 0.090 & 0.139 \\
\cline { 2 - 11 } & 4.0 & 0.421 & 0.310 & 0.024 & 0.221 & 1.6 & 0.492 & 0.195 & 0.073 & 0.139 \\
\cline { 2 - 11 } & 4.5 & 0.454 & 0.270 & 0.024 & 0.193 & 1.9 & 0.498 & 0.195 & 0.061 & 0.139 \\
\cline { 2 - 10 } & 5.0 & 0.480 & 0.239 & 0.023 & 0.171 & 2.2 & 0.502 & 0.195 & 0.053 & 0.139 \\
\hline \multirow{4}{*}{.3} & 3.0 & 0.284 & 0.439 & 0.079 & 0.313 & 1.0 & 0.345 & 0.195 & 0.351 & 0.139 \\
\cline { 2 - 10 } & 3.5 & 0.349 & 0.363 & 0.076 & 0.259 & 1.3 & 0.388 & 0.195 & 0.270 & 0.139 \\
\cline { 2 - 10 } & 4.0 & 0.395 & 0.310 & 0.074 & 0.221 & 1.6 & 0.414 & 0.195 & 0.219 & 0.139 \\
\cline { 2 - 10 } & 4.5 & 0.429 & 0.270 & 0.073 & 0.193 & 1.9 & 0.433 & 0.195 & 0.185 & 0.139 \\
\cline { 2 - 10 } & 5.0 & 0.455 & 0.239 & 0.071 & 0.171 & 2.2 & 0.446 & 0.195 & 0.159 & 0.139 \\
\hline \hline
\end{tabular}

\section{(c) Effect of Failure Rate and Repair Rate}

The effect of $\alpha(\beta)$ on the various performance characteristics can be seen in Table 3. We see an increasing (decreasing) trend in $\mathrm{P}_{\mathrm{E}}, \mathrm{P}_{\mathrm{O}}$ and $\mathrm{P}_{\mathrm{D}}$ whereas decreasing (increasing) trend in $P_{V}$ with the increase in $\alpha(\beta)$.

Table 3. Effect of $p, \alpha$ and $\beta$ on various performance characteristics.

\begin{tabular}{|c||c||c|c|c|c||c||c|c|c|c||}
\hline $\mathbf{p}$ & $\alpha$ & $\mathbf{P}_{\mathbf{V}}$ & $\mathbf{P}_{\mathbf{E}}$ & $\mathbf{P}_{\mathbf{O}}$ & $\mathbf{P}_{\mathbf{D}}$ & $\boldsymbol{\beta}$ & $\mathbf{P}_{\mathbf{V}}$ & $\mathbf{P}_{\mathbf{E}}$ & $\mathbf{P}_{\mathbf{O}}$ & $\mathbf{P}_{\mathbf{D}}$ \\
\hline \multirow{4}{*}{.1} & 0.70 & 0.484 & 0.204 & 0.024 & 0.204 & 3.0 & 0.630 & 0.234 & 0.028 & 0.035 \\
\cline { 2 - 11 } & 0.75 & 0.475 & 0.206 & 0.024 & 0.221 & 3.5 & 0.646 & 0.228 & 0.027 & 0.031 \\
\cline { 2 - 11 } & 0.80 & 0.466 & 0.209 & 0.025 & 0.239 & 4.0 & 0.663 & 0.223 & 0.026 & 0.027 \\
\cline { 2 - 10 } & 0.85 & 0.457 & 0.211 & 0.025 & 0.257 & 4.5 & 0.679 & 0.219 & 0.026 & 0.025 \\
\cline { 2 - 10 } & 0.90 & 0.447 & 0.214 & 0.025 & 0.275 & 5.0 & 0.697 & 0.214 & 0.025 & 0.023 \\
\hline \hline \multirow{4}{*}{.3} & 0.70 & 0.458 & 0.204 & 0.073 & 0.204 & 3.0 & 0.603 & 0.234 & 0.084 & 0.035 \\
\cline { 2 - 10 } & 0.75 & 0.449 & 0.206 & 0.074 & 0.221 & 3.5 & 0.619 & 0.228 & 0.082 & 0.031 \\
\cline { 2 - 10 } & 0.80 & 0.440 & 0.209 & 0.075 & 0.239 & 4.0 & 0.635 & 0.223 & 0.080 & 0.027 \\
\cline { 2 - 10 } & 0.85 & 0.430 & 0.211 & 0.076 & 0.257 & 4.5 & 0.651 & 0.219 & 0.078 & 0.025 \\
\cline { 2 - 10 } & 0.90 & 0.420 & 0.214 & 0.077 & 0.275 & 5.0 & 0.667 & 0.214 & 0.077 & 0.023 \\
\hline
\end{tabular}


(d) Comparison of Exact Waiting Time with Approximate Waiting Time

Tables 4-5 are constructed to examine the comparison between $\mathrm{W}_{\mathrm{q}}$ and $\hat{W}_{q}$ by varying the parameters $\lambda, \mu_{1}, \mu_{2}, \alpha, \beta$ and $\theta$ for the two different values of $\mathrm{p}$. It is clear from the tables that $\mathrm{W}_{\mathrm{q}}$ and $\hat{W}_{q}$ reveal the increasing (decreasing) trend for the increasing values of $\lambda$ and $\alpha\left(\mu_{1}, \mu_{2}\right.$, $\theta$ and $\beta$ ) for both values of $p$. Further, it can be noticed from Tables 4-5 that absolute percentage error (APE) varies from $0 \%-18 \%$ which is reasonably less.

Table 4. Comparison between exact and approximate waiting time by varying $\lambda$, $\mu_{1}$ and $\mu_{2}$.

\begin{tabular}{|c|c|c|c|c|c|c|c|}
\hline \multicolumn{2}{|c|}{} & \multicolumn{3}{c|}{$\mathbf{p}=\mathbf{0 . 5}$} & \multicolumn{3}{|c|}{$\mathbf{p}=\mathbf{0 . 6}$} \\
\hline \hline \multirow{2}{*}{ Parameters } & $\mathbf{W}_{\mathbf{q}}$ & $\hat{W}_{q}$ & $\mathbf{A P E}$ & $\mathbf{W}_{\mathbf{q}}$ & $\hat{W}_{q}$ & \multirow{2}{*}{ APE(\%) } \\
\hline \multirow{4}{*}{$\lambda$} & 1.20 & 4.22 & 4.75 & 2.58 & 5.03 & 4.72 & 6.15 \\
\cline { 2 - 9 } & 1.21 & 4.50 & 4.83 & 7.21 & 5.34 & 4.79 & 10.2 \\
\cline { 2 - 9 } & 1.22 & 4.80 & 4.91 & 2.33 & 5.66 & 4.86 & 14.1 \\
\cline { 2 - 9 } & 1.23 & 5.10 & 4.99 & 2.10 & 5.99 & 4.93 & 17.6 \\
\cline { 2 - 9 } & 1.24 & 5.41 & 5.07 & 6.20 & 6.33 & 5.00 & 20.9 \\
\hline \multirow{4}{*}{$\mu_{1}$} & 2.12 & 4.49 & 4.76 & 6.02 & 5.36 & 4.74 & 11.4 \\
\cline { 2 - 8 } & 2.14 & 4.30 & 4.69 & 9.01 & 5.17 & 4.69 & 9.23 \\
\cline { 2 - 8 } & 2.16 & 4.13 & 4.63 & 12.0 & 4.98 & 4.63 & 6.91 \\
\cline { 2 - 8 } & 2.18 & 3.96 & 4.56 & 15.2 & 4.80 & 4.58 & 4.54 \\
\hline \multirow{4}{*}{$\mu_{2}$} & 2.20 & 3.79 & 4.50 & 18.6 & 4.62 & 4.52 & 2.11 \\
\cline { 2 - 8 } & 1.94 & 5.46 & 5.14 & 5.85 & 6.37 & 5.07 & 20.35 \\
\cline { 2 - 8 } & 1.95 & 5.39 & 5.13 & 4.80 & 6.29 & 5.07 & 19.43 \\
\cline { 2 - 8 } & 1.96 & 5.33 & 5.13 & 3.74 & 6.22 & 5.07 & 18.50 \\
\cline { 2 - 8 } & 1.97 & 5.26 & 5.12 & 2.68 & 6.15 & 5.07 & 17.57 \\
\hline
\end{tabular}

Table 5. Comparison between exact and approximate waiting time by varying $\alpha, \beta$ and $\theta$.

\begin{tabular}{|c|c|c|c|c|c|c|c|}
\hline \multicolumn{2}{|c|}{} & \multicolumn{3}{c|}{$\mathbf{p = 0 . 5}$} & \multicolumn{3}{c|}{$\mathbf{p = 0 . 6}$} \\
\hline \hline \multicolumn{2}{|c|}{ Parameters } & $\mathbf{W}_{\mathbf{q}}$ & $\hat{W}_{q}$ & $\mathbf{A P E}(\%)$ & $\mathbf{W}_{\mathbf{q}}$ & $\hat{W}_{q}$ & APE(\%) \\
\hline \multirow{4}{*}{$\alpha$} & 0.22 & 7.62 & 7.50 & 1.49 & 8.63 & 7.30 & 15.4 \\
\cline { 2 - 8 } & 0.24 & 8.09 & 8.16 & 0.91 & 9.11 & 7.92 & 13.0 \\
\cline { 2 - 8 } & 0.26 & 8.52 & 8.81 & 3.36 & 9.56 & 8.54 & 10.6 \\
\cline { 2 - 8 } & 0.28 & 8.93 & 9.46 & 5.86 & 9.98 & 9.15 & 8.30 \\
\cline { 2 - 8 } & 0.30 & 9.32 & 10.1 & 8.43 & 10.3 & 9.76 & 5.86 \\
\hline \multirow{4}{*}{$\beta$} & 0.11 & 11.8 & 13.4 & 13.5 & 14.0 & 13.4 & 4.31 \\
\cline { 2 - 8 } & 0.12 & 10.2 & 11.2 & 8.85 & 12.1 & 11.1 & 8.05 \\
\cline { 2 - 8 } & 0.13 & 9.01 & 9.42 & 4.60 & 10.5 & 9.35 & 11.5 \\
\cline { 2 - 8 } & 0.14 & 7.96 & 8.01 & 0.65 & 9.32 & 7.93 & 14.8 \\
\hline \multirow{4}{*}{$\boldsymbol{N}$} & 0.96 & 5.55 & 5.18 & 6.62 & 6.17 & 5.18 & 15.9 \\
\cline { 2 - 8 } & 0.97 & 5.37 & 5.19 & 3.29 & 6.06 & 5.20 & 14.1 \\
\cline { 2 - 8 } & 0.98 & 5.20 & 5.20 & 0.01 & 5.95 & 5.22 & 12.3 \\
\cline { 2 - 8 } & 0.99 & 5.04 & 5.21 & 3.21 & 5.85 & 5.23 & 10.5 \\
\cline { 2 - 8 } & 1.00 & 4.89 & 5.21 & 6.41 & 5.74 & 5.24 & 8.79 \\
\hline
\end{tabular}




\section{Average queue length}

Figures 2-4 are plotted for the queue length against $\lambda, \theta, \mu_{1}, \mu_{2}, \alpha$ and $\beta$ for various values of $p$. From Fig. 2, we see that initially there is a sharp increment (decrement) in the average queue length with the increasing values of $\lambda(\theta)$ and it also increases (decreases) with $p$. On the other hand from Fig. 3, we observe that on increasing the service rates ( $\mu_{1}$ and $\left.\mu_{2}\right)$, there is the decreasing trend in $\mathrm{E}\left(\mathrm{N}_{\mathrm{q}}\right)$. It is quite visible from Fig. 4 that queue length first decreases (increases) slowly then after smoothly for increasing values of repair rate (breakdown rate).

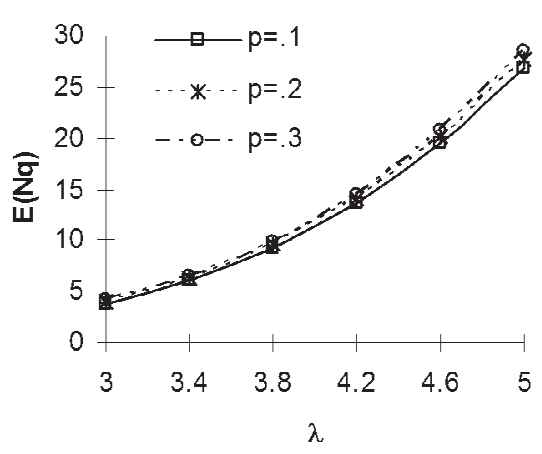

2(a)

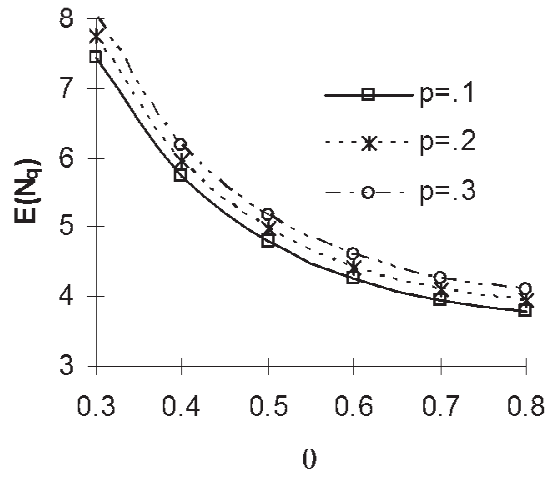

2(b)

Fig. 2. $\mathbf{E}\left(\mathbf{N}_{\mathbf{q}}\right)$ vs. (a) $\lambda$ and (b) $\theta$, for different values of $\mathbf{p}$.

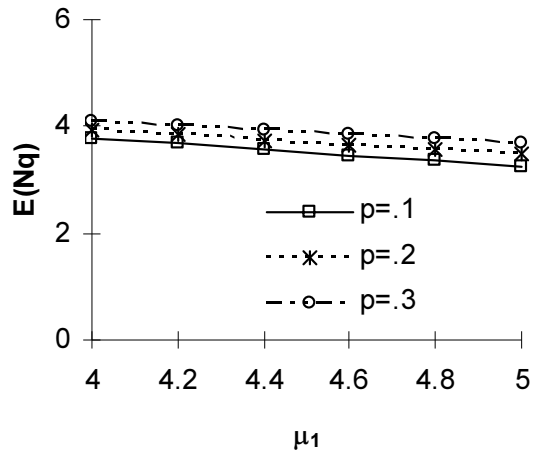

3(a)

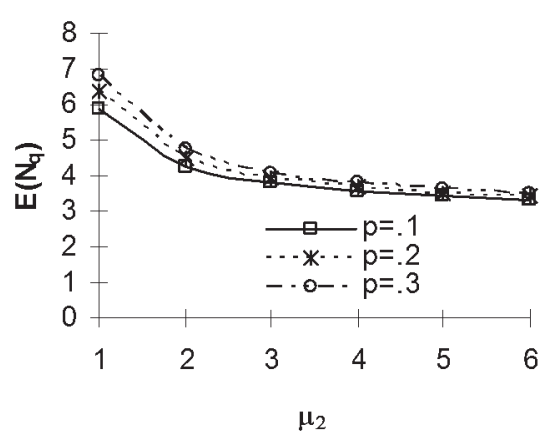

3(b)

Fig. 3. $E\left(N_{q}\right)$ vs. (a) $\mu_{1}$ and (b) $\mu_{2}$ for different values of $p$. 


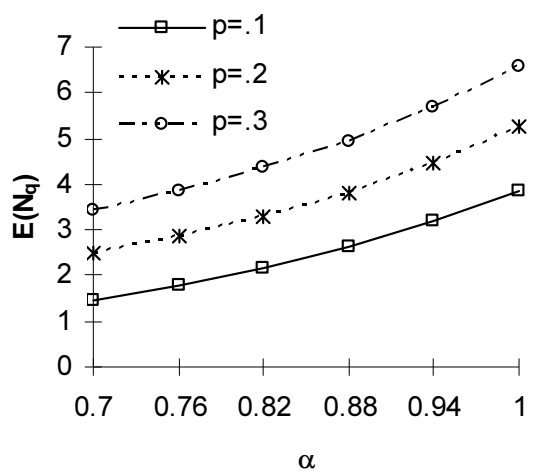

4(a)

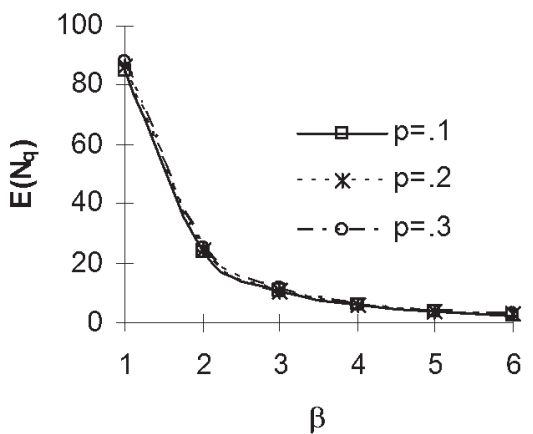

4(b)

Fig. 4. $E\left(N_{q}\right)$ vs. (a) $\alpha$ and (b) $\beta$ for different values of $p$.

From all tables and graphs, we conclude that

The queue length and the waiting time of the customers can be reduced by increasing the service rate which matches with many realistic situations.

As expected, the queue length increases gradually then sharply with arrival rate, vacation rate and breakdown rate.

APE between the exact and the approximate waiting times of the queue length is found to be reasonably less. So, MEA provides the good approximate results for the exact results which are obtained by using the analytical method.

\section{Conclusions}

In this investigation, the concept of maximum entropy is employed to analyze the probability distribution for the number of the customers in different states of the server for an unreliable server $\mathrm{M} / \mathrm{M} / 1$ queue with vacation and additional phase of optional service renders in batch of fixed size. The concepts of server breakdown along with vacation and optional bulk service considered may be useful for the queueing systems wherein the unreliable server may intend to utilize the idle time for rest or some other tasks. Numerical illustrations and sensitivity analysis performed to examine the effect of different parameters on various system characteristics provides an insight for optimal control of system 
descriptors. It is hoped that our study will be helpful to deal with congestion problems encountered in many industrial scenarios such as manufacturing system, inventory system, computer system, etc. The present study can be further extended by including the concepts of $\mathrm{N}$ policy and bulk arrival but the analysis will be more cumbersome and tedious.

\section{References}

[1] Doshi, B.T., Queueing systems with vacations: A survey, Queueing Systems, 1: 29-66 (1986).

[2] Labzovski, S.N., Mehrez, A. and Frenkel, I.B., The a priori vacation probability in the M/G/1 single vacation models, Math. Comp. Simul., 54(1-3): 183-188 (2000).

[3] Zhang, Z.G., On the convexity of the two-threshold policy for an $\mathrm{M} / \mathrm{G} / 1$ queue with vacations, Oper. Res. Letter., 34(4): 473-476 (2006).

[4] Choudhury, G., Tadj, L. and Paul, M., Steady state analysis of an $\mathrm{M}^{\mathrm{x}} / \mathrm{G} / 1$ queue with two phases service and Bernoulli vacation under multiple vacation policy, Appl. Math. Model., 31(6): 1079-1091 (2007).

[5] Choudhury, G., A single server queueing system with two phases of service and vacations, Qual. Tech. Quant. Manage., 5(1): 33-49 (2008).

[6] Chae., K.C., Lim, D.E. and Yang, W.S., The GI/M/1 queue and the GI/Geo/1 queue both with single working vacation, Perform. Eval., 66(7): 356-367 (2009).

[7] Goswami, C. and Selvaraju, N., The discrete-time MAP/PH/1 queue with multiple working vacations, Appl. Math. Model., 34(4): 931-946 (2010).

[8] Jain, M., Sharma, G.C. and Sharma, R., $\mathrm{M}^{\mathrm{r}} / \mathrm{M}^{(\mathrm{a}, \mathrm{d}, \mathrm{b})} / 1$ queue with state dependent bulk service accessible and non-accessible batches, Oper. Res., Information Technology and Industry, M. Jain \& G. C. Sharma (ed.). Anamaya Publishers, New Delhi (2002).

[9] Gupta, U.C. and Sikdar, K., The finite-buffer M/G/1 queue with general bulk-service rule and single vacation, Perform. Eval., 57: 199-219 (2004).

[10] Sikdar, K. and Gupta, U.C., Analytical and numerical aspects of batch service queues with single vacation, Comp. Oper. Res., 32(4): 943-966 (2005).

[11] [Al-khedhairi, A. and Tadj, L., A bulk service queue with choice of service and re-service under Bernoulli schedule, Int. J. Contemp. Math. Sci., 2(23): 1107-1120 (2007).

[12] Gupta, U.C. and Sikdar, K., On the batch arrival batch service queue with finite buffer under server's vacation: $\mathrm{M}^{\mathrm{X}} / \mathrm{G}^{\mathrm{Y}} / 1 / \mathrm{N}$ queue, Comp. Math. Appl., 56(11): 2861-2873 (2008).

[13] Murugan, S.P.B. and Kalyanaraman, R., A vacation queue with additional optional service in batches, Appl. Math. Sci., 3(24): 1203-1208 (2009).

[14] Lenin, R.B., Cuyt, A. Yoshigoe, K. and Ramaswamy, S., Computing packet loss probabilities of D-BMAP/PH/1/N queues with group services, Perform. Eval., 67(3): 160173 (2010).

[15] Gray, W.J., Wang, P.P. and Scott, M., A vacation queueing model with server breakdowns, Appl. Math. Model., 24(5-6): 391-400 (2000).

[16] Ke, J. C., Modified T vacation policy for an $\mathrm{M} / \mathrm{G} / 1$ queueing system with an unreliable server and startup, Math. Comput. Model., 41(11-12): 267-1277 (2005).

[17] Ke, J.C., On M/G/1 system under NT policies with breakdowns, startup and closedown, Appl. Math. Model., 30(1): 49-66 (2006).

[18] Fiems, D. Maertens, T. and Bruneel, H., Queueing systems with different types of server interruptions, Euro. J. Oper. Res., 188(3): 838-845 (2008).

[19] Choudhury, G., Tadj, L. and Deka, K., A batch arrival retrial queueing system with two phases of service and service interruption, Comp. Math. Appl., 59(1): 437-450 (2010). 
[20] Economou, A. and Kapodistria, S., Synchronized abandonments in a single server unreliable queue, Euro. J. Oper. Res., 203(1): 143-155 (2010).

[21] Efrosinin, D. and Winkler, A., Queueing system with a constant retrial rate, non-reliable server and threshold-based recovery, Euro. J. Oper. Res., 210(3): 594-605 (2011).

[22] Wang, K.H., Wang, T.Y. and Pearn, W.L., Maximum entropy analysis to the N-policy M/G/1 queueing system with server breakdowns and general startup times, Appl. Math. Comput., 165: 45-61 (2005).

[23] Ke, J.C. and Lin, C.H., Maximum entropy solutions for batch arrival queue with an unreliable server and delaying vacations, Appl. Math. Comput., 183(2): 1328-1340 (2006).

[24] Ke, J.C. and Lin, C.H., Maximum entropy approach for batch-arrival queue under N policy with an un-reliable server and single vacation, J. Comput. Appl. Math., 221(1): 1-15 (2008).

[25] Wang, K.H., Chan, M.C. and Ke, J. C., Maximum entropy analysis of the $\mathrm{M}^{[\mathrm{x}]} / \mathrm{M} / 1$ queueing system with multiple vacations and server breakdowns, Comput. Indust. Engg., 52(2): 192-202 (2007).

[26] Wang, K.H. and Huang, K.B., A maximum entropy approach for the (p,N)-policy M/G/1 queue with a removable and unreliable server, Appl. Math. Model., 33(4): 2024-2034 (2009).

[27] Madan, K.C., An M/M/1 queueing system with additional optional service in batches, IAPQR Transacations, 17(1) 23-33 (1992).

[28] Gross, D. and Harris, C. M., Fundamentals of Queueing Theory, $2^{\text {nd }}$ ed., John Wiley and Sons, New York: (1985).

[29] El-Affendi, M.A. and Kouvatsos, D.D., A maximum entropy analysis of the M/G/1 and G/M/1 queueing systems at equilibrium, Acta Info., 19: 339-355 (1983). 


\section{أسلوب الحد الأقصى للإنتروبي في حالة من عدم مصداقية خادم في عطلة في نموذج الصفوف مع اختيار لتكدس الخدمة}

\section{مادهي جان، و *ج. س. شارما، و*** ريشا شارما}

قسم الرياصيات، دعهُ روركي التكنولوجي الهندي، و" معهـ العلوم الأساسية،

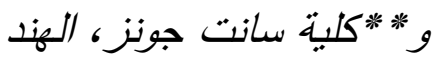

الهستخلص. في هذه الدراسة تم الأخذ في الاعتبار وجود خادم واحد

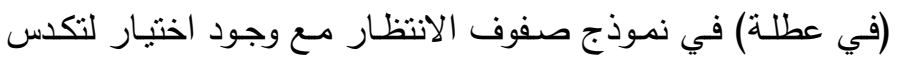

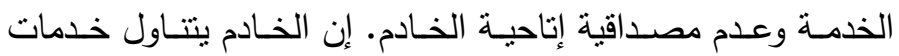

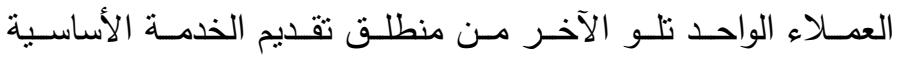

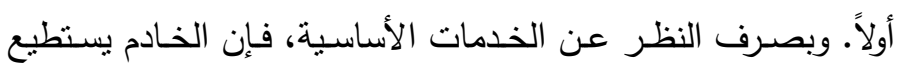

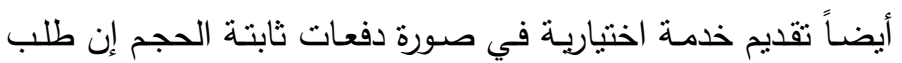

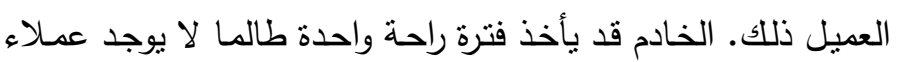

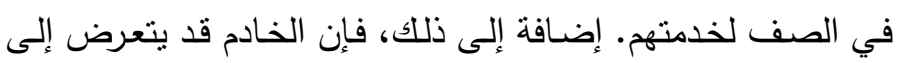

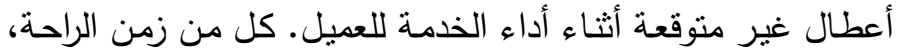

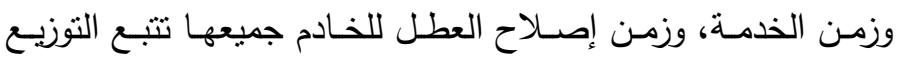

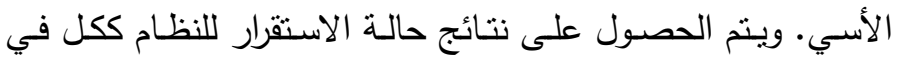

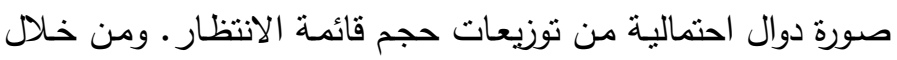

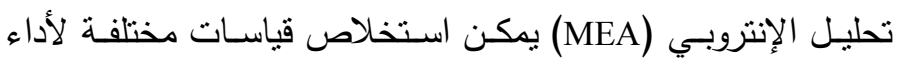

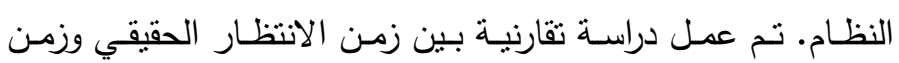

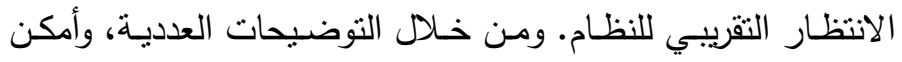

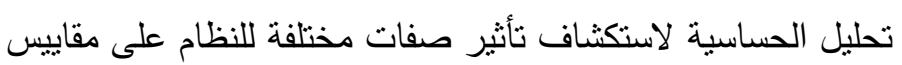

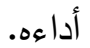

